\title{
Design and Synthesis of a Novel Tetrapeptide with Improved Selectivity Towards the hMC1R
}

\author{
Jonathon R. Sawyer, Saghar Mowlazadeh Haghighi, Yang Zhou, \\ Kaitlyn McLeod, Victor J. Hruby, and Minying Cai \\ Department of Chemistry and Biochemistry, University of Arizona, Tucson, AZ, 85721, USA
}

\section{Introduction}

The melanocortin system plays vital roles with regard to maintaining homeostasis. The endogenous melanotropin peptides $(\alpha-, \beta-, \gamma$-melanocyte stimulating hormones $(\mathrm{MSH})$ are derived from the proopiomelanocortin (POMC) preprohormone gene. These melanotropin peptides all contain the same core pharmacophore amino acid sequence: His-Phe-Arg-Trp [1,2]. This sequence is important for binding to the five human melanocortin receptors (MC1R, MC2R, MC3R, MC4R, and MC5R). Binding of the endogenous melanotropin peptides to these melanocortin receptors results in several responses depending on which receptor is activated. Such responses involve feeding behavior, learning behavior, pain modulation, pigmentation, and sexual function. The primary native ligand for all five of the melanocortin receptors is $\alpha-\mathrm{MSH}$. Unfortunately, $\alpha$-MSH along with the other endogenous melanotropin peptides do not possess high selectivity for any of the melanocortin receptors as well as are biologically unstable. Thus, the quest to discover potent, biologically stable, and highly selective peptides began.

\section{Results and Discussion}

Peptide Design. Previous research led to a breakthrough discovery of a more biologically stable and potent derivative of $\alpha-\mathrm{MSH}$ as seen in NDP- $\alpha-\mathrm{MSH}$ (MT-I) with the key modifications of 4-norleucine and 7-D-phenylalanine [3]. Currently, MT-1 is a marketed drug under the name of Afamelanotide for the treatment of congenital erythropoietic porphyria. However, MT-I does not have high selectivity to any one of the melanocortin receptor subtypes, which can lead to unexpected side effects. Because the tetrapeptide pharmacophore sequence of NDP- $\alpha-\mathrm{MSH}$ is necessary for binding affinity to the melanocortin receptors but with poorer potency and selectivity to all subtypes of hMCRs, modifying the tetrapeptide structure will be of critical importance to improve the potency and the selectivity. Our earlier studies demonstrated that the electrostatic interaction, $\operatorname{Arg}(\mathrm{L})-\operatorname{Asp}(\mathrm{R})$, between the $\operatorname{Arg}^{8}$ of the NDP- $\alpha-$ MSH and the Asp122, Asp126 of the hMC4R is of critical importance to achieve binding and receptor activation [4]. Similarly, a key interaction between the $\mathrm{Arg}^{8}$ of the NDP-MSH and the D154 as well as the D158 of the MC3R is necessary for binding [5]. Therefore, switching the arginine in the tetrapeptide to a neutrally charged amino acid, in particular norleucine, should reduce binding towards the hMC3R and the hMC4R. We envision that enhanced selectivity towards the MC1R can be reached with reduced electrostatic interaction between the $\operatorname{Arg}^{8}(\mathrm{~L})-\operatorname{Asp}(\mathrm{R})$ of the tetrapeptide and the respective aspartic acids on the MC3R and MC4R receptors.

NDP- $\alpha$-MSH (MT-I) has been well developed with potent binding and cAMP activities towards all subtypes of hMCRs (Figure 1). However, it does not have selectivity to hMCRs. Earlier studies demonstrated that the pharmacophore of NDP- $\alpha-\mathrm{MSH}$ is His-D-Phe-Arg-Trp, a tetrapeptide shown here as peptide 1, which maintains binding as well as cAMP activities to all subtypes of hMCRs but with much lower potency and efficacy. To improve the selectivity of hMCRs, herein we made a leading peptide 2, which has replaced $\mathrm{Arg}^{8}$ with $\mathrm{Nle}^{8}$. Our biological studies demonstrate that peptide $\mathbf{2}$ has cAMP activity and selectivity towards the hMC1R, weak activity at the hMC4R (ten orders of magnitude weaker), and no functional cAMP activity at the hMC3R and the hMC5R (Table 1). The observed result indicates that changing the $\mathrm{Arg}^{8}$ to $\mathrm{Nle}^{8}$ in the peptide 2 improves the functional selectivity of hMC1R. Hence, abolishing the positive charge of the amino acid in the tetrapeptide, His-D-Phe-Arg-Trp, improves selectivity towards the hMC1R. Further research, which involves structural modifications of peptide $\mathbf{2}$, is going on in order to produce a more potent and selective MC1R agonist/antagonist activity. 


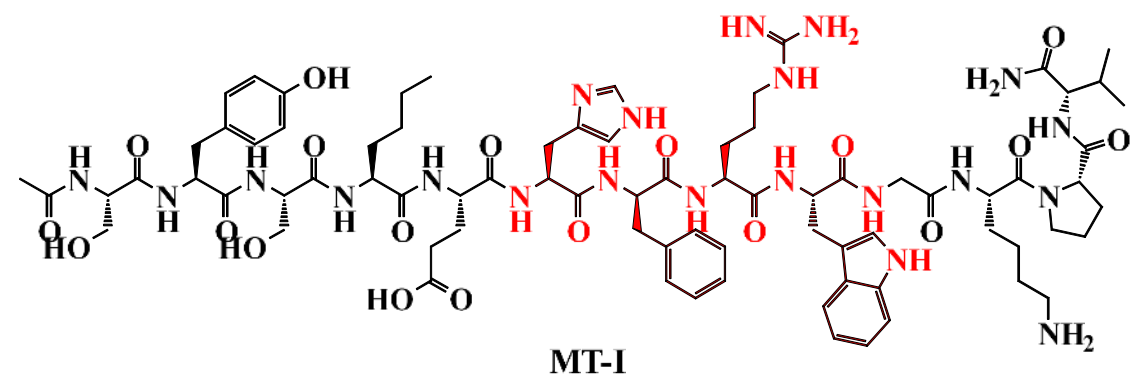<smiles>CCCC[C@H](NC(=O)[C@H](Cc1c[nH]c2ccccc12)NC(=O)[C@H](Cc1ccccc1)NC(=O)[C@H](Cc1c[nH]cn1)NC(C)=O)C(=O)N[C@@H](Cc1c[nH]c2ccccc12)C(N)=O</smiles>

Fig. 1. Chemical structures of MT-1 (known as NDP- $\alpha-M S H)$, tetrapeptide (1), and the MC1R selective peptide (2).

Peptide Synthesis. Standard solid-phase methods were used. Fmoc chemistry was used and the peptide was synthesized on Rink Amide resin. The resin was first deprotected using 20\% (v/v) piperidine in DMF solution followed by coupling of the first amino acid. The Kaiser Test was performed to ensure that coupling went to completion. Once it was determined that coupling was complete, the growing peptide chain was deprotected using $20 \%(\mathrm{v} / \mathrm{v})$ piperidine in DMF solution followed by coupling of the second amino acid. The remaining amino acids were added to the growing peptide chain in the same manner. Each coupling reaction included the amino acid, HCTU, and DIPEA. After the final amino acid was coupled, the $N$-terminus was acetylated. The peptide was cleaved from the resin and all side-chain protecting groups were removed using the cleavage cocktail of $95 \%$ TFA, $2.5 \%$ TIS, and $2.5 \%$ water.

Table 1. cAMP Assays of MT-I (known as NDP- $\alpha-M S H)$, tetrapeptide 1, MC1R selective peptide 2, and MT-II towards Melanocortin Receptors.

\begin{tabular}{ccccccccc}
\hline & \multicolumn{2}{c}{ MC1R } & \multicolumn{2}{c}{ MC3R } & \multicolumn{2}{c}{$M C 4 R$} & \multicolumn{2}{c}{$M C 5 R$} \\
& \multicolumn{2}{c}{$c A M P$} & \multicolumn{2}{c}{$c A M P$} & \multicolumn{2}{c}{$c A M P$} & \multicolumn{2}{c}{$c A M P$} \\
& $E C_{50}$ & $\%$ Act & $E C_{50}$ & $\%$ Act & $E C_{50}$ & $\%$ Act & $E C_{50}$ & $\%$ Act \\
\hline MT-1 & - & 100 & $0.8 \pm 0.3$ & 100 & $0.2 \pm 0.04$ & 100 & $1 \pm 0.3$ & 100 \\
$\mathbf{1}$ & 4.5 & 100 & 7.1 & 100 & 607 & 100 & 2788 & 35 \\
$\mathbf{2}$ & 261 & 58 & $\mathrm{NA}$ & 0 & 2874 & 100 & $\mathrm{NA}$ & 0 \\
MT-II & $1.02 \pm 0.4$ & 100 & $5.1 \pm 0.3$ & 100 & $2.1 \pm 0.6$ & 100 & $5.7 \pm 2.2$ & 100 \\
\hline
\end{tabular}

${ }^{a} M T-I I=A c-N l e-c\left[\right.$ Asp-His-D-Phe-Arg-Trp-Lys]-NH2. EC $C_{50}=$ Effective concentration of peptide that was able to generate $50 \%$ maximal intracellular cAMP accumulation $(N=4)$. Act $\%=\%$ of cAMP produced at $10 \mu M$ ligand concentration, in relation to MT-II. NA $=0 \%$ cAMP accumulation observed at $10 \mu M$. The peptides were tested at a range of concentration from $10^{-10}$ to $10^{-5} \mathrm{M}$. 


\section{Acknowledgements}

Supported in part by a grant from the U.S. Public Health Service, National Institutes of Health, DK017420, GM 108040 and DA06284.

\section{References}

1. Hruby, V.J. Nat. Rev. Drug Discov. 1, 847-858 (2002), http://dx.doi.org/10.1038/nrd939

2. Hruby, V.J., Wilkes, B.C., Hadley, M.E., Al-Obeidi, F., Sawyer, T.K., Staples, D.J., deVaux, A.E., Dym, O., Castrucci, A., Hintz, M.F., Riehm, J.P., Rao, K.R. J. Med. Chem. 30, 2126-2130 (1987), http://dx.doi.org/10.1021/jm00394a033

3. Sawyer, T.K., Sanfilippo, P.J., Hruby, V.J., Engel, M.H., Heward, C.B., Burnett, J.B., Hadley, M.E. Proc.

Natl. Acad. Sci. USA 77, 5754-5758 (1980), http://dx.doi.org/10.1073/pnas.77.10.5754

4. Chen, M., Cai, M., Aprahamian, C.J., Georgeson, K.E., Hruby, V., Harmon, C.M., Yang, Y. J. Biol. Chem. 282, 21712-21719 (2007), http://dx.doi.org/10.1074/jbc.M702285200

5. Chen, M., Aprahamian, C.J., Celik, A., Georgeson, K.E., Garvey, W.T., Harmon, C.M., Yang, Y. Biochemistry 130, 1128-1137 (2006). http://dx.doi.org/10.1021/bi0521792 